\title{
REVIEW
}

\section{Medical therapy for spermatogenic failure}

\author{
Ranjith Ramasamy, Peter J Stahl and Peter N Schlegel
}

Medical treatment of men with primary spermatogenic failure remains largely ineffective in contrast to those with secondary testicular failure. Treatment has been attempted with a multitude of agents ranging from hormones to nutritional supplements (antioxidants). While some studies have demonstrated benefit to some treatments, no treatments have consistently demonstrated efficacy nor has it been possible to reliably identify patients likely to benefit. Idiopathic spermatogenic failure likely results from multiple discrete defects in sperm production that are as yet unidentified. A better understanding of these defects will yield more effective treatment options and appropriate triage of patients to specific therapeutic regimens. This review focuses on the rationale and current evidence for hormonal and antioxidant therapy in medical treatment of male infertility, spermatogenic failure in particular. Although empiric medical therapy for spermatogenic failure has been largely replaced by assisted reproductive techniques, both treatment modalities could play a role, perhaps as combination therapy.

Asian Journal of Andrology (2012) 14, 57-60; doi:10.1038/aja.2011.63; published online 19 December 2011

Keywords: antioxidant; hormones; male infertility; sperm; spermatogenesis; spermatogenic failure; testicular failure

\section{INTRODUCTION}

Testicular failure is characterized by impairment of the endocrine (testosterone (T) production) and/or exocrine (sperm production) functions of the testis. The phenotype of primary exocrine testicular failure is impaired spermatogenesis leading to male infertility. Spermatogenic failure may result from hypothalamic, pituitary or testicular disorders. Specific and effective medical treatment is available for men with gonadotropin deficiencies, but such patients are rare. The more common variant of severe male infertility is primary testicular failure, which is characterized by normal or low serum $\mathrm{T}$ and elevated or normal serum follicle-stimulating hormone (FSH) levels. In most of these men, no specific cause of spermatogenic failure that could be targeted by medical therapy is identifiable. As a consequence, various empirical medical treatments are often used to treat idiopathic male infertility, with limited success. Specific medical therapy for low serum $\mathrm{T}$ levels associated with increased aromatase activity (relatively elevated estradiol levels) has also been reported. A comprehensive workup for men with infertility using semen analyses, hormonal studies, genetic and radiological evaluation is necessary in order to identify men who will benefit from medical therapy and assisted reproductive technologies should be reserved for those who do not respond favorably.

\section{HORMONAL TREATMENT}

\section{Gonadotropins}

A low serum FSH, luteinizing hormone and $\mathrm{T}$ indicate hypogonadotropic hypogonadism $(\mathrm{HH})$ that may be idiopathic $(\mathrm{IHH})$, or due to congenital gonadotropin-releasing hormone $(\mathrm{GnRH})$ deficiency (e.g., Kallmann's syndrome) or due to identifiable lesions in the hypothalamo-pituitary axis including or a wide range of acquired causations (pituitary adenoma, other central nervous system tumors, systemic diseases such as hemochromatoisis and sarcoidosis). In some cases, diagnosis and treatment of these underlying conditions may improve testicular function while also providing additional health benefits.

Spermatogenesis may be restored in most HH men with gonadotropin replacement therapy. ${ }^{1}$ Gonadotropin therapy and pulsatile GnRH are highly effective in inducing spermatogenesis in men with $\mathrm{HH}$. The two therapies do not appear to differ in the time to first appearance of sperm, sperm densities or pregnancy rates; ${ }^{2,3}$ however, because of the cumbersome nature of wearing a continuous infusion pump (subcutaneous), few centers use pulsatile GnRH therapy.

Gonadotropin deficiency is conventionally treated with human chorionic gonadotropin (hCG) (as an luteinizing hormone substitute; 1500-2000 IU 2-3 times a week subcutaneously) alone or combined with FSH in the form of menopausal gonadotropin (75 IU subcutaneously 2-3 times weekly) or recombinant human FSH (100-150 IU 2-3 times weekly) (rhFSH). Typically, luteinizing hormone deficiency is corrected first by administration of hCG, the dose being adjusted to achieve a nadir T level measured $48 \mathrm{~h}$ after hCG injection in the midnormal range. If after 4-6 months of hCG, no sperm is detected in the semen, supplemental treatment with recombinant or highly purified human FSH is initiated. ${ }^{4}$ This regimen may take up to $1-2$ years to achieve its maximum effect on sperm production.

The efficacy of combination treatment with rhFSH and hCG to restore fertility in men with $\mathrm{HH}$ is supported by substantial clinical evidence. In one prospective observational study, average testicular volume increased from 4.1 to $12.4 \mathrm{ml}$ and the mean total motile sperm count increased from zero to 4.8 million. ${ }^{5}$ Men with postpubertal onset of $\mathrm{HH}$ are more likely to respond to hCG alone with re-initiation of spermatogenesis than those with prepubertal onset who are likely to require addition of $\mathrm{FSH}^{6}$ 
Warne et al. ${ }^{4}$ showed that rhFSH combined with hCG is effective for the restoration of fertility in the majority of men with $\mathrm{HH}$. In a study of 81 men with $\mathrm{HH}$, who remained azoospermic but achieved normal serum T concentrations after hCG pretreatment, 68 (84.0\%) achieved spermatogenesis and $56(69.1 \%)$ achieved spermatozoa concentrations $\geqslant 1.5 \times 10^{6} \mathrm{ml}^{-1}$. They identified that men with large baseline mean testicular volume, low body mass index and advanced sexual maturity were predictors of good response to therapy. In another smaller study with 21 men, Matsumoto et al. ${ }^{7}$ showed that long-term treatment of azoospermic $\mathrm{HH}$ men using rhFSH and hCG is effective for stimulating spermatogenesis and is well-tolerated. ${ }^{7}$

In 2003, a multicenter, open-label, phase III, randomized efficacy and safety study ${ }^{8}$ was performed with combined hCG and rhFSH treatment to induce spermatogenesis in men with $\mathrm{HH}$. A weekly dose of $450 \mathrm{IU}(3 \times 150$ or $2 \times 225 \mathrm{IU}) \mathrm{rhFSH}$, in addition to hCG, was able to induce spermatogenesis in many hypogonadotropic azoospermic men who failed to respond to treatment with hCG alone. Treatment time necessary for first sperm cells to appear in the ejaculate was related to the initial testicular volume.

The best predictors of response to gonadotropin therapy are testicular volume and time of onset of gonadotropin deficiency (pre- or postpubertal). ${ }^{9,10}$ Those with testicular volumes greater than $8 \mathrm{ml}$, reflecting less severe gonadotropin deficiency, and those with postpubertal onset of gonadotropin deficiency are more likely to respond than those with testicular volumes less than $4 \mathrm{ml}$ and prepubertal onset. ${ }^{9,10}$ Cryptorchidism requiring orchidopexy has a negative prognostic effect on spermatogenic induction in IHH. ${ }^{11}$ Even the limited testicular volume seen in men with $\mathrm{IHH}$, it is interesting to speculate whether hCG used during puberty may induce a greater spermatogenic potential and testicular growth.

Pulsatile GnRH administration can be typically started with an initial dose of $25 \mathrm{ng} \mathrm{kg}^{-1}$ per pulse administered subcutaneously every $2 \mathrm{~h}$ by a portable infusion pump. ${ }^{10}$ The dose of GnRH is adjusted until the serum $\mathrm{T}$ level is in the mid-normal range. Doses ranging from 25 to $200 \mathrm{ng} \mathrm{kg}^{-1}$ may be required to induce virilization. ${ }^{10}$ After successful induction of secondary sex characteristics, the GnRH dose can be reduced. Gonadal function can be maintained in a majority of $\mathrm{HH}$ men by pulsatile GnRH therapy. ${ }^{10}$

In men without demonstrable $\mathrm{HH}$, there is little evidence to support the use of gonadotropin therapy. However, there is some rationale and limited evidence for the use of rhFSH in some situations. One clinical trial suggested that administration of rhFSH may be effective in a subset of patients with idiopathic oligoasthenozoospermia. Foresta et al. randomized 112 oliogoasthenozoospermic patients to treatment with $100 \mathrm{U}$ of rhFSH every other day for 3 months or non-treatment. ${ }^{12}$ Overall, the treated cohort showed no benefit. However, a subgroup analysis identified a group of treatment responders in whom seminal parameters improved and the unassisted pregnancy rate was significantly better than the nonresponders or the untreated group. The responders were patients who had cytological evidence of hypospermatogenesis on a fine needle aspiration. While the majority of men with primary testicular failure have elevated $\mathrm{FSH}$, some men with spermatogenic failure such as those with maturation arrest histology can also have normal FSH and might be candidates for empirical therapy, but this ought be considered experimental until evidence from further placebocontrolled trials can provide supporting evidence.

FSH receptor gene polymorphisms have been studied as potential risk factors for spermatogenetic failure and may ultimately enable selection of men with primary testicular failure who are more likely to benefit from rhFSH therapy. Selice et al. ${ }^{13}$ evaluated the response of FSH treatment in terms of sperm production on the basis of polymorphisms in the FSH receptor and showed that only subjects with at least one serine in position 680 showed a statistically significant increase in these sperm parameters. The analysis of this gene could represent a pharmacogenetic approach to the treatment of male infertility, but this approach must be validated by other investigators prior to being incorporated into clinical practice.

\section{Dopamine receptor agonists}

If a prolactin-secreting pituitary macro- or micro-adenoma is identified during evaluation of $\mathrm{HH}$, then medical treatment with a dopamine receptor agonist is indicated. Cabergoline $(0.125-1.0 \mathrm{mg}$ twice weekly) is the preferred agent because it has the highest efficacy in normalizing prolactin levels and shrinking prolactin-secreting tumors, though bromocriptine is less expensive and also effective despite a higher risk of side effects. Reversal of infertility with dopamine agonist therapy occurs in $53 \%$ of cases. ${ }^{14}$

\section{Anti-estrogens}

Before the introduction of intracytoplasmic sperm injection, treatment options for idiopathic male factor subfertility were mainly based on anti-estrogens. Anti-estrogen therapy has been popular due to the safety, low cost and ease of administration. Clomiphene and tamoxifen are estrogen receptor modulators with predominant antagonist activity that block negative feedback exerted by estrogen at the hypothalamus and anterior pituitary. The result is increased pituitary gonadotropin production that could stimulate both testicular production of $\mathrm{T}$ and spermatogenesis.

A meta-analysis evaluated 738 subfertile males with oligoasthenozoospermia who were exposed to short-term treatment protocols by anti-estrogens. ${ }^{15}$ The pregnancy rate was $15.4 \%$ in the patient group versus $12.5 \%$ in the control group (odds ratio: 1.56 ; $95 \%$ CI: $0.99-$ 2.19). The authors concluded that there is no enough evidence to support the value of anti-estrogens in the management of oligoasthenozoospermia. Unfortunately, many relevant well-designed clinical trials of clomiphene and tamoxifen for male subfertility have been performed in combination with other agents. The efficacy of each agent alone is not established. A randomized controlled trial ${ }^{16}$ of daily clomiphene $(25 \mathrm{mg}$ ) plus vitamin E (400 mg) in men with idiopathic oligoasthenozoospermia demonstrated the efficacy of this regimen over placebo in improving total sperm count, forward progressive motility, and unassisted pregnancy rate $(37 \%$ versus $13 \%, P=0.04)$ but only involved 30 couples in each limb with borderline significant odds ratio for pregnancy (odds ratio: 3.76; 95\% CI: 1.03-13.64). In a case series from three international centers, comprising 42 patients with non-obstructive azoospermia, $64 \%$ of patients responded to clomiphene citrate and produced sperm in the ejaculate, sufficient for intracytoplasmic sperm injection. The study group included cases of maturation arrest (43\%) and hypospermatogenesis (57\%), and no cases of Sertoli cell-only syndrome. The dose of clomiphene citrate was titrated to achieve a serum $\mathrm{T}$ level between 600 and $800 \mathrm{ng} \mathrm{dl}^{-1}$ (20-24 $\left.\mathrm{nmol} \mathrm{l}^{-1}\right)$. The post-treatment sperm concentration ranged from 1 to 16 million sperm/ml (mean: 3.8). ${ }^{17}$ Since no control group was evaluated, no treatment-related effect on sperm concentration can be established.

\section{Aromatase inhibitors}

Aromatase inhibitors decrease the conversion of the androgens ( $\mathrm{T}$ and androstenedione) to estrogens (estradiol and estrone), thereby 
increasing serum androgen levels. The mechanism of increasing $\mathrm{T}$ is likely by decreasing feedback inhibition of pituitary and hypothalamus, effecting greater gonadotropin release. ${ }^{18}$ Administration of aromatase inhibitors such as testolactone and anastrozole restores the $\mathrm{T} / \mathrm{E}_{2}$ ratio to normal and has been suggested to significantly improve semen parameters in oligozoospermic men, including sperm concentration and motility. ${ }^{18,19}$ However, neither of these studies was a placebo-controlled randomized study design, but was rather single open limb design, one involving subgroups selected with arbitrarily defined 'high E/T' ratios. Fertile men have mean $\mathrm{T}\left(\mathrm{ng} \mathrm{dl}^{-1}\right) / \mathrm{E}_{2}\left(\mathrm{pg} \mathrm{ml}^{-1}\right)$ ratios of $14.5 \pm 1.2$, whereas men with non-obstructive azoospermia have a $\mathrm{T} / \mathrm{E}_{2}$ ratios of $6.9 \pm 0.6^{19}$ and men with Klinefelter's syndrome a $\mathrm{T} / \mathrm{E}_{2}$ ratio of $4.4 \pm 0.5 .{ }^{18}$ Therefore, it appears that at least a small subset of male infertility patients with elevated serum estradiol levels may benefit from treatment with an aromatase inhibitor (anastrazole $1 \mathrm{mg}$ daily, testalactone $100-200 \mathrm{mg}$ daily or other aromatase inhibitors). ${ }^{18}$ Candidates for aromatase inhibition have usually been identified as men with serum T/E ratios $<10 .{ }^{19}$ Men with Klinefelter's syndrome seem to benefit more from the administration of testolactone rather than anastrozole; ${ }^{20}$ however, testolactone is not commercially available in the United States. The effectiveness of aromatase inhibition suggests that aromatase activity from Leydig cell hyperplasia may be responsible for increased $\mathrm{T}$ to estradiol conversion and impaired semen parameters. ${ }^{18}$

\section{ANTIOXIDANT THERAPY}

Infertile men have higher levels of seminal reactive oxygen species than fertile men. ${ }^{21}$ High levels of semen reactive oxygen species are associated sperm dysfunction, sperm DNA damage and reduced male reproductive potential. This observation has led clinicians to treat infertile men with antioxidant supplements. A variety of clinical studies, the majority of which are poorly designed, suggest that dietary antioxidant supplements are beneficial in terms of improving sperm function and DNA integrity. ${ }^{22-24}$ However, the exact mechanism of action of dietary antioxidants and the optimal dietary supplement has not been established. ${ }^{25}$ There are no placebo-controlled trials that have demonstrated a significantly higher unassisted pregnancy rate when the male partner of a subfertile couple is treated with empiric antioxidant therapy. ${ }^{5,26}$ A beneficial effect of in vitro antioxidant supplements in protecting spermatozoa from exogenous oxidants has been demonstrated in most studies; however, the benefits, if any of these antioxidants in protecting sperm from endogenous reactive oxygen species, gentle sperm processing and cryopreservation, have not been conclusively established. ${ }^{27,28}$

Interestingly, a recent Cochrane Collaboration meta-analysis ${ }^{29}$ showed statistically significant four to fivefold increases in the pregnancy and live birth rates among subfertile men using assisted reproduction who are treated with antioxidants. No such meta-analysis has been done for antioxidant therapy of subfertile men attempting to conceive naturally. Unfortunately, this meta-analysis could not identify the specific agents or dosage to recommend for treatment of infertile men. Current literature suggests that carnitines and vitamin $\mathrm{C}$ and $\mathrm{E}$ have been shown to be effective for improvements in semen analysis parameters by many well-conducted studies and may be considered as a treatment option. ${ }^{30-32}$ A systematic review ${ }^{33}$ analyzed 17 randomized trials, including a total of 1665 men who were treated with antioxidants. Despite the methodological and clinical heterogeneity, 14 of the 17 (82\%) trials showed an improvement in either sperm quality or pregnancy rate after antioxidant therapy. Six of 10 trials showed a significant improvement in pregnancy rate after antioxidant therapy. ${ }^{33}$ There is, however, a need for further investigation with randomized controlled studies to confirm the efficacy and safety of antioxidant supplementation in the medical treatment of spermatogenic failure (decreased sperm count) as well as the need to determine the ideal dose of each compound to improve semen parameters, fertilization rates and pregnancy outcomes. Choices of therapy should therefore be based upon physician experience and patient preference. Although the beneficial effect on fertility remains to be established, zinc, folate and herbal remedies ${ }^{22,34-36}$ are used by patients and practitioners alike to improve semen quality. These supplements need to be used with caution until evidence is clearly established.

\section{CONCLUSION}

In many subfertile couples, there are no identifiable female factors and either no modifiable male factors are identifiable, or subfertility persists despite treatment of an identified male factor. Medical treatment could enhance natural conception or improve outcomes with assisted reproduction. However, medical therapy should not be used in patients with known genetic factors such as karyotype anomalies or Y chromosome deletion. Therefore, it is essential to perform a complete diagnostic workup of the male before deciding on which men will respond to medical therapy and those who need to be referred to assisted reproduction. Couples who elect to proceed with empiric medical treatment must be counseled that such treatment may be ineffective and could lead to delays in assisted reproduction that may adversely affect outcome.

\section{COMPETING FINANCIAL INTERESTS}

The authors have no financial interests relevant to the subject matter of this manuscript.

1 Sokol RZ. Endocrinology of male infertility: evaluation and treatment. Semin Reprod Med 2009; 27: 149-58.

2 Kliesch S, Behre HM, Nieschlag E. High efficacy of gonadotropin or pulsatile gonadotropin-releasing hormone treatment in hypogonadotropic hypogonadal men. Eur J Endocrinol 1994; 131: 347-54.

3 Liu L, Chaudhari N, Corle D, Sherins RJ. Comparison of pulsatile subcutaneous gonadotropin-releasing hormone and exogenous gonadotropins in the treatment of men with isolated hypogonadotropic hypogonadism. Fertil Steril 1988; 49: 302 8.

4 Warne DW, Decosterd G, Okada H, Yano Y, Koide N et al. A combined analysis of data to identify predictive factors for spermatogenesis in men with hypogonadotropic hypogonadism treated with recombinant human follicle-stimulating hormone and human chorionic gonadotropin. Fertil Steril 2009; 92: 594-604.

5 Saleh RA, Agarwal A. Oxidative stress and male infertility: from research bench to clinical practice. J Androl 2002; 23: 737-52.

6 Bhasin S. Approach to the infertile man. J Clin Endocrinol Metab 2007; 92: 1995 2004.

7 Matsumoto AM, Snyder PJ, Bhasin S, Martin K, Weber T et al. Stimulation of spermatogenesis with recombinant human follicle-stimulating hormone (follitropin alfa; GONAL-f): long-term treatment in azoospermic men with hypogonadotropic hypogonadism. Fertil Steril 2009; 92: 979-90.

8 Bouloux PM, Nieschlag E, Burger HG, Skakkebaek NE, Wu FC et al. Induction of spermatogenesis by recombinant follicle-stimulating hormone (puregon) in hypogonadotropic azoospermic men who failed to respond to human chorionic gonadotropin alone. J Androl 2003; 24: 604-11.

9 Liu PY, Gebski VJ, Turner L, Conway AJ, Wishart SM et al. Predicting pregnancy and spermatogenesis by survival analysis during gonadotrophin treatment of gonadotrophin-deficient infertile men. Hum Reprod 2002; 17: 625-33.

10 Spratt DI, Finkelstein JS, O'Dea LS, Badger TM, Rao PN et al. Long-term administration of gonadotropin-releasing hormone in men with idiopathic hypogonadotropic hypogonadism. A model for studies of the hormone's physiologic effects. Ann Intern Med 1986; 105: 848-55.

11 Pitteloud N, Hayes FJ, Dwyer A, Boepple PA, Lee H et al. Predictors of outcome of longterm $\mathrm{GnRH}$ therapy in men with idiopathic hypogonadotropic hypogonadism. J Clin Endocrinol Metab 2002; 87: 4128-36 
Medical treatment for male infertility

R Ramasamy et al.

60

12 Foresta C, Bettella A, Garolla A, Ambrosini G, Ferlin A. Treatment of male idiopathic infertility with recombinant human follicle-stimulating hormone: a prospective, controlled, randomized clinical study. Fertil Steril 2005; 84: 654-61.

13 Selice R, Garolla A, Pengo M, Caretta N, Ferlin A et al. The response to FSH treatment in oligozoospermic men depends on FSH receptor gene polymorphisms. Int J Androl 2011; 34: 306-12.

14 Melmed S, Casanueva FF, Hoffman AR, Kleinberg DL, Montori VM et al. Diagnosis and treatment of hyperprolactinemia: an Endocrine Society clinical practice guideline. J Clii Endocrinol Metal 2011; 96: 273-88.

15 Vandekerckhove P, Lilford R, Vail A, Hughes E. Clomiphene or tamoxifen for idiopathic oligo/asthenospermia. Cochrane Database Syst Rev 2000; (2): CD000151.

16 Ghanem H, Sheer O, El-Segini A. Combination clomiphene citrate and antioxidant therapy for idiopathic male infertility: a randomized controlled trial. Fertil Steril 2010; 93: 2232-5.

17 Hussein A, Ozgok Y, Ross L, Niederberger C. Clomiphene administration for cases of nonobstructive azoospermia: a multicenter study. J Androl 2005; 26: 787-91; discussion 92-3.

18 Roman JD, Schlegel PN. Aromatase inhibitors for male infertility. J Urol 2002; 167: 624-9.

19 Pavlovich CP, King P, Goldstein M, Schlegel PN. Evidence of a treatable endocrinopathy in infertile men. J Urol 2001; 165: 837-41.

20 Ramasamy R, Ricci JA, Palermo GD, Gosden LV, Rosenwaks Z et al. Successful fertility treatment for Klinefelter's syndrome. J Ural 2009; 182: 1108-13.

21 Abd-Elmoaty MA, Sale R, Sharma R, Agarwal A. Increased levels of oxidants and reduced antioxidants in semen of infertile men with varicocele. Fertil Steril 2010; 94 : 1531-4.

22 Safarinejad MR, Safarinejad S. Efficacy of selenium and/or $\mathrm{N}$-acetyl-cysteine for improving semen parameters in infertile men: a double-blind, placebo controlled, randomized study. J Ural 2009; 181: 741-51.

23 Om AE, Al-Azemi MK, Kehinde EO, Anim JT, Oriowo MA et al. Indications of the mechanisms involved in improved sperm parameters by zinc therapy. Med Princ Pact 2008; 17: 108-16.
24 Scott R, MacPherson A, Yates RW, Hussain B, Dixon J. The effect of oral selenium supplementation on human sperm motility. Br J Ural 1998; 82: 76-80.

25 Zini A, Al-Hathal N. Antioxidant therapy in male infertility: fact or fiction? Asian J Androl 2011; 13: 374-81.

26 Zini A, Phillips S, Lefebvre J, Baazeem A, Bissonnette F et al. Anti-sperm antibodies are not associated with sperm DNA damage: a prospective study of infertile men. J Reprod Immunol 2009; 85: 205-8.

27 Lopes S, Jurisicova A, Sun JG, Casper RF. Reactive oxygen species: potential cause for DNA fragmentation in human spermatozoa. Hum Reprod 1998; 13: 896-900.

28 Russo A, Troncoso N, Sanchez F, Garbarino JA, Vanilla A. Propolis protects human spermatozoa from DNA damage caused by benzo[a]pyrene and exogenous reactive oxygen species. Life Sci 2006; 78: 1401-6.

29 Showell MG, Brown J, Yazdani A, Stankiewicz MT, Hart RJ. Antioxidants for male subfertility. Cochrane Database Syst Rev 2011; (1): CD007411.

30 Agarwal A, Sekhon LH. The role of antioxidant therapy in the treatment of male infertility. Hum Fertil (Camb) 2010; 13: 217-25.

31 Costa M, Candle D, Filicori M, D'Lddio S, Lenzi A. L-carnitine in idiopathic asthenozoospermia: a multicenter study. Italian Study Group on Carnitine and Male Infertility. Andrologia 1994; 26: 155-9.

32 Dawson EB, Harris WA, Peter MC, Powell LC. Effect of ascorbic acid supplementation on the sperm quality of smokers. Fertil Steril 1992; 58: 1034-9.

33 Ross C, Morris A, Khairy M, Khalaf Y, Braude P et al. A systematic review of the effect of oral antioxidants on male infertility. Reprod Boomed Online 2010; 20: 711-23.

34 Wong WY, Merkus HM, Thomas CM, Menkveld R, Zielhuis GA et al. Effects of folic acid and zinc sulfate on male factor subfertility: a double-blind, randomized, placebocontrolled trial. Fertil Steril 2002; 77: 491-8.

35 Safarinejad MR. Effect of pentoxifylline on semen parameters, reproductive hormones, and seminal plasma antioxidant capacity in men with idiopathic infertility: a randomized double-blind placebo-controlled study. Int Ural Nephrol 2011; 43: 315-28.

36 Ko EY, Sabanegh ES Jr. The role of over the counter supplements for the treatment of male infertility - fact or fiction? J Androl; e-pub ahead of print 19 May 2011; doi:10.2164/jandrol.111.013730.

Asian Journal of Andrology 